\title{
Diversidad e infectividad de hongos micorrícicos arbusculares nativos provenientes de algarrobales del Parque Chaqueño argentino con características edafoclimáticas contrastantes
}

Sagadin, M. B., Monteoliva, M. I., Luna C. M. y Cabello M. N.

\begin{abstract}
RESUMEN
En ambientes severos, la presencia de hongos micorrícicos arbusculares (HMA) es clave para la supervivencia de las plantas y el balance ecosistémico. El objetivo de este trabajo fue caracterizar los hongos micorrícicos arbusculares presentes en sitios de algarrobales (Prosopis alba) del Parque Chaqueño argentino de condiciones climáticas contrastantes: húmedo, Colonia Benítez, y semiárido, Padre Lozano. Los sitios de Colonia Benítez y Padre Lozano presentaron diferencias edafológicas significativas, destacándose el menor contenido de materia orgánica y fósforo, y el mayor porcentaje de sodio intercambiable en Colonia Benítez, asociados a una mayor capacidad infectiva y mayor potencial micorrícico de inóculo. En los suelos sólo se encontró una especie común: Acaulospora laevis, propia de ambientes áridos. En los inóculos se registraron tres especies en común: Funneliformis mosseae, Rhizophagus intraradices, Claroideoglomus etunicatum; dos especies exclusivas de Colonia Benítez, Septoglomus constrictum y Rhizophagus clarus; y dos especies exclusivas de Padre Lozano, Claroideoglomus claroideum y Diversispora spurca. Este es el primer estudio en Argentina en el cual se reporta la diversidad de HMA en suelos de algarrobales y se mide la infectividad de los suelos y de los inóculos obtenidos a partir de esos suelos.
\end{abstract}

Palabras clave: Hongos micorrícicos arbusculares, algarrobales, Parque Chaqueño, infectividad, potencial micorrícico, diversidad.

Sagadin, M. B., Monteoliva, M. I., Luna C. M. and Cabello M. N., 2018. Diversity and infectivity of native arbuscular mycorrhizal fungi from Prosopis alba areas with contrasting edaphoclimatic characteristics in the Argentinian Parque Chaqueño. Agriscientia 35 (2): 19-33 


\section{SUMMARY}

In severe environments, the presence of arbuscular mycorrhizal fungi (AMF) is essential for plant survival and a balanced ecosystem e. The aim of this work was to characterize the arbuscular mycorrhizal fungi present in algarrobales (Prosopis alba) areas of contrasting humid and semi-arid climatic conditions, Colonia Benítez and Padre Lozano respectively, both in the Parque Chaqueño region in Argentina. The Colonia Benítez and Padre Lozano sites showed significant edaphological differences, the most important being the lower content of organic matter and phosphorus, and the higher percentage of exchangeable sodium in Colonia Benítez, associated with a higher infective capacity and greater mycorrhizal potential of inoculum. Only one species was found in both soils: Acaulospora laevis, typical of arid environments. In the inocula, three species were identified in both sites: Funneliformis mosseae, Rhizophagus intraradices, Claroideoglomus etunicatum; two species were only identified in Colonia Benítez: Septoglomus constrictum and Rhizophagus clarus; and two species were only identified in Padre Lozano: Claroideoglomus claroideum and Diversispora spurca. This is the first study in Argentina to analyze AMF diversity in Prosopis alba areas (algarrobales) and their infectivity in the soils and their inocula.

Key words: Arbuscular mycorrhizal fungi, Prosopis alba areas, Parque Chaqueño, infectivity, mycorrhizal potential, diversity.

Sagadin, M. B.: Instituto de Fisiología y Recursos Genéticos Vegetales, Centro de Investigaciones Agropecuarias (IFRGV-CIAP), Instituto Nacional de Tecnología Agropecuaria (INTA), Camino de las 60 Cuadras Km 5.5, X5020ICA. Córdoba, Argentina. Monteoliva, M. I. y Luna, C. M.: Instituto de Fisiología y Recursos Genéticos Vegetales, Centro de Investigaciones Agropecuarias (IFRGV-CIAP), Instituto Nacional de Tecnología Agropecuaria (INTA). Consejo de Investigaciones Científicas y Técnicas de la República Argentina (CONICET). Cabello M. N.: Instituto de Botánica Carlos Spegazzini, Facultad de Ciencias Naturales y Museo, Universidad Nacional de La Plata, B1900AVJ. La Plata, Buenos Aires, Argentina. Comisión de Investigaciones Científicas (CIC), La Paz, Buenos Aires, Argentina. Correspondencia a: sagadin.monica@inta.gob.ar

\section{INTRODUCCIÓN}

El Parque Chaqueño ocupa la región central norte de Argentina donde las altas temperaturas y la sequía afectan el establecimiento y el desarrollo de los bosques de algarrobo implantados (López Lauenstein et al., 2012). Prosopis alba Griseb. es una de las especies nativas de mayor valor comercial en Argentina por su multiplicidad de usos, desde la utilización de la madera en la fabricación de muebles o como combustible, hasta el uso como alimento para el hombre y el ganado. Debido a sus cualidades, este recurso ha sido sobreexplotado y la producción de plantines de calidad es un requisito indispensable para asegurar la supervivencia y el adecuado desarrollo de los bosques implantados (Salifu y Jacobs, 2006).
Los HMA, al establecer una asociación simbiótica con las raíces de las plantas, favorecen una eficiente explotación de las fuentes minerales y del agua del suelo, constituyendo una herramienta de utilidad en la sobrevida y adaptación de las especies vegetales. La capacidad de las micorrizas para promover el desarrollo de los cultivos depende de dos factores: efectividad e infectividad. La efectividad se refiere al potencial que tienen los HMA para desarrollar la simbiosis micorrícica e incrementar el crecimiento del hospedero. Y la infectividad se refiere a la capacidad del hongo para penetrar e invadir la raíz intensamente y explorar el suelo, así como su habilidad de persistir en el sistema productivo (Tapia-Goné et al., 2010).

En ambientes severos donde las condiciones son críticas para otros microorganismos, la 
presencia de los HMA es considerada clave para la supervivencia de las plantas y el balance ecosistémico. En algunos casos, la adaptación entre los HMA y las plantas hospedantes está determinada por las condiciones edafoclimáticas, mostrando diferencias estructurales y funcionales entre especies e incluso entre morfotipos dentro de la misma especie de hongo (Maherali y Klironomos, 2007). El conocimiento del potencial micorrícico de los suelos y su infectividad, junto con su efectividad para la micorrización, es muy importante para el estudio de la ecología y el manejo de estos hongos (Herrera-Peraza, Hamel, Fernández, Ferrer y Furrazola, 2011).

La selección de microorganismos nativos de una región brinda mayores posibilidades de adaptación y multiplicación de los mismos en el suelo, debido a que la diversidad funcional de los HMA puede depender de la procedencia de los aislamientos más que de la especie fúngica (Trejo et al., 2011). En tal sentido, se ha reportado que los HMA nativos de ambientes semiáridos incorporan agua y nutrientes más eficientemente en suelos secos, confiriéndole a la planta una mayor resistencia a la sequía. Además, la utilización de inóculos nativos mixtos (que contengan tres o más especies de HMA) es de interés en ensayos de restauración de áreas degradadas, tanto por su mayor adaptación a las condiciones imperantes en el ecosistema, como por la mayor diversidad de respuestas potenciales que varias especies de HMA pueden ejercer sobre una planta hospedera. Particularmente las esporas pueden identificarse y complementar la evaluación de la diversidad de especies locales en diferentes ecosistemas. El establecimiento de cultivos trampa utilizando suelo a granel, o mezclando suelo y piezas de raíz y haciéndolos crecer con huéspedes micotróficos, representa una buena estrategia para producir una gran cantidad de esporas saludables. Sin embargo, esta metodología no necesariamente permite la identificación de todas las especies, debido a que la esporulación de la comunidad de hongos puede verse afectada por la planta trampa elegida, mientras en otros casos puede promover la esporulación de especies de HMA crípticas que no estaban presentes en el tiempo de muestreo o condiciones de campo.

En Argentina se han observado HMA nativos en diversas regiones: en trigo (Cabello, 2013), en leguminosas y en gramíneas forrajeras, tales como agropiro y festuca (Cabello, 2013; Covacevich, Marino y Echeverría, 2006). Sin embargo, existen pocos estudios sobre la diversidad de HMA nativos en zonas de algarrobales (Piatti e Iglesias, 2004) donde sólo se ha reportado la colonización micorrícica, pero no se identificaron las especies de HMA.

Por lo expuesto, los objetivos de este estudio fueron: 1) estimar la infectividad de los HMA presentes en dos sitios de algarrobales bajo condiciones edafoclimáticas contrastantes (húmedo y semiárido del Parque Chaqueño); 2) evaluar el potencial micorrícico de inóculos generados en plantas trampa a partir de los suelos contrastantes; 3) identificar las especies de HMA presentes en los suelos y en los inóculos. La hipótesis de este trabajo es que las características edafoclimáticas condicionan la diversidad e infectividad de los HMA nativos de sitios de algarrobales, potencialmente benéficos para la simbiosis con Prosopis alba.

\section{MATERIALES Y MÉTODOS}

\section{Selección de los sitios, área de muestreo y recolección de las muestras de suelo}

Las muestras de suelo fueron colectadas en primavera, entre fines de noviembre y mediados de diciembre, de dos sitios del Parque Chaqueño argentino de rodales puros de Prosopis alba Griseb. (Figura 1): Colonia Benítez (CB) 27 20' 00" $\mathrm{S}, 58^{\circ} 55^{\prime} 60^{\prime \prime} \mathrm{O}$, de la provincia de Chaco, con un régimen pluviométrico anual de $1300 \mathrm{~mm}$; y Padre Lozano (PL) $23^{\circ} 12^{\prime} 51^{\prime \prime} \mathrm{S}, 63^{\circ} 50^{\prime} 39^{\prime \prime} \mathrm{O}$, NE de la provincia de Salta, con un régimen pluviométrico anual de $650 \mathrm{~mm}$ (Cabrera, 1976).

El sitio de Padre Lozano pertenece a la región Chaco Semiárido de la provincia de Salta (Figura 1). El clima es de tipo semiárido, con una precipitación media anual de $650 \mathrm{~mm}$ y gran variación interanual en los registros pluviométricos. Las precipitaciones se concentran en verano y la evapotranspiración potencial genera déficit hídrico todo el año. Desde un enfoque fitogeográfico, la región se ubica en el distrito Chaqueño Occidental, correspondiente a un bosque xerófilo caducifolio con dos especies dominantes: quebracho colorado (Schinopsis quebracho-colorado) y quebracho blanco (Aspidosperma quebracho-blanco), acompañados en un segundo estrato de mistol (Zizyphus mistol), algarrobos (Prosopis alba, P. nigra, P. elata, P. torcuata), guayacán (Caesalpinia paraguariensis) y yuchán (Ceiba insignis), y con un tercer estrato arbustivo. El estrato herbáceo ha desaparecido debido a la presión del ganado, que desencadena procesos de desertificación (Camardelli, Kirschbaum, Kirschbaum y Fabrezi, 2015). El sitio de Colonia Benítez pertenece a la localidad homónima de la provincia del Chaco, se encuentra en la región oriental, recibe 

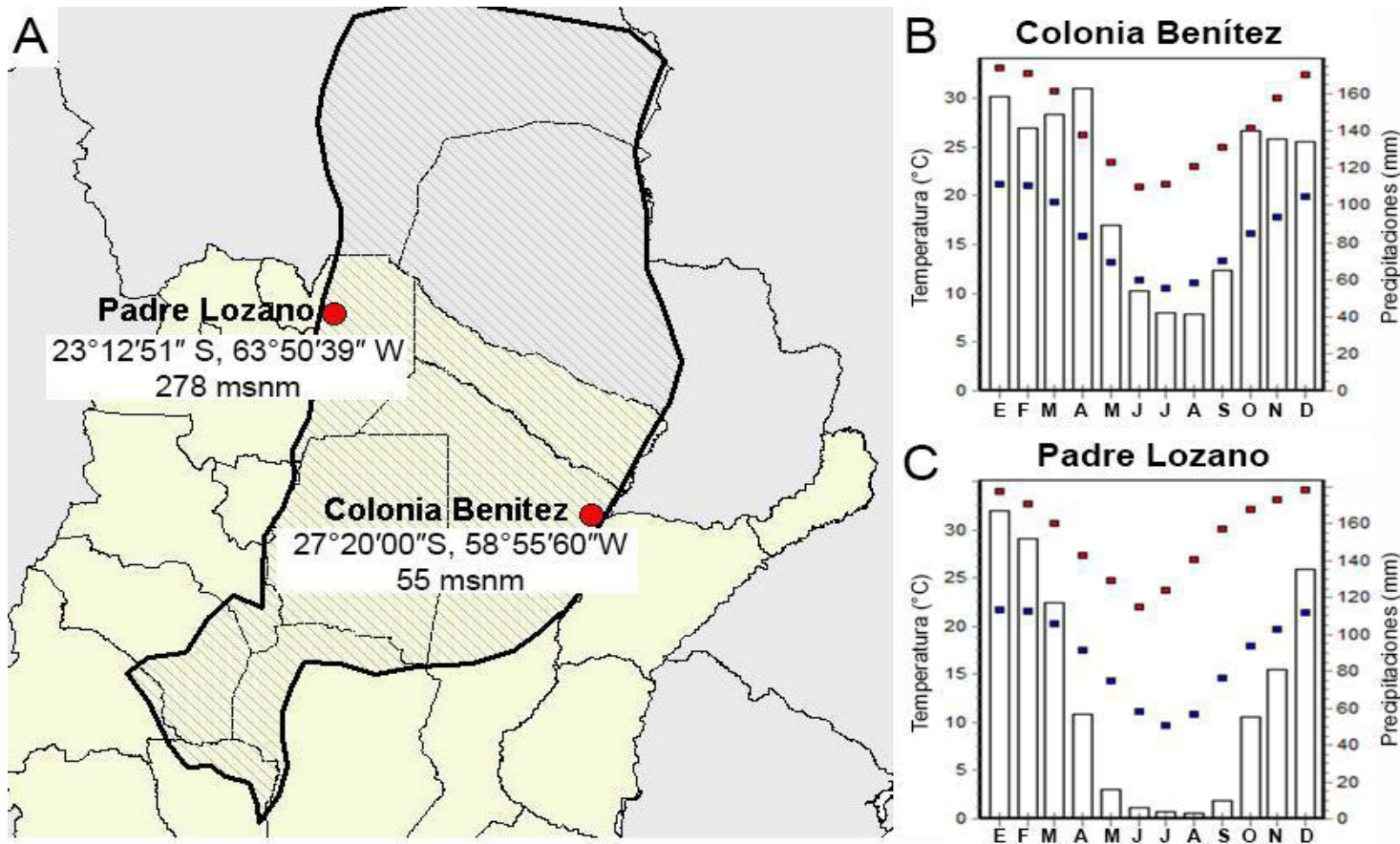

Figura 1. Ubicación geográfica (A) y climograma (b) de los sitios de muestreo Colonia Benítez (húmedo) (B) y Padre Lozano (semiárido) (C) en la región chaqueña de Argentina. Se muestran las temperaturas máximas y mínimas (cuadrados rojos y azules respectivamente) y precipitaciones medias mensuales de cada sitio como barras blancas. Los datos fueron tomados de la base de datos World Clim (Hijmans et al., 2005).

precipitaciones abundantes que promedian los $1300 \mathrm{~mm}$ anuales. Se caracteriza por un paisaje salpicado de esteros, lagunas, bañados y zonas anegadizas. Los suelos de esta región pueden definirse, a grandes rasgos, como sedimentarios de origen fluvial y lacustre, formados por material fino como arcilla, arena y limos. La relación entre las temperaturas y las precipitaciones es un factor decisivo para el desarrollo de la vegetación. En Eco-regiones de la Argentina (Rodríguez, 2018), se clasificó a Colonia Benítez como Chaco Húmedo. En esta ecorregión la comunidad florística más importante la constituyen los quebrachales, con predominio del quebracho colorado chaqueño (S. balansae) y, en menor cantidad, el quebracho blanco (A. quebracho-blanco), de menor porte que el anterior. A estos se les suma el guayacán (C. paraguariensis), el algarrobo negro ( $P$. nigra), el algarrobo blanco (P. alba) y el mistol (Z. mistol) entre muchas otras especies (Rodríguez, 2018).

De un rodal puro de Prosopis alba, se tomó una hectárea de cada sitio dentro de la cual se eligieron cinco árboles de algarrobo equidistantes entre sí (100 m, aproximadamente). El muestreo se realizó luego de eliminar la capa superficial del material no descompuesto, y fueron obtenidas a una profundidad entre 0 y $20 \mathrm{~cm}$; las muestras se sacaron debajo de la proyección de la copa de los árboles. Se tomaron seis submuestras por cada árbol conformando una muestra homogénea única por árbol (Becerra y Cabello, 2008; Arévalo Hernández, 2016) la cual fue mantenida a $4{ }^{\circ} \mathrm{C}$ hasta su utilización. Posteriormente fueron secadas a temperatura ambiente y tamizadas con malla de $2 \mathrm{~mm}$. Parte de las muestras fueron enviadas para su análisis físico-químico al Laboratorio de Suelos de la Facultad de Ciencias Agropecuarias de la Universidad Nacional de Córdoba. Para aislar e identificar las esporas presentes en el suelo de los dos sitios, se tomaron tres muestras de $50 \mathrm{~g}$ de suelo por sitio.

De cada sitio muestreado se determinó: contenido de fósforo extractable (Bray y Kurtz, 1945), nitrógeno (Bremmer, 1965), carbono orgánico (Walkley y Black, 1934), fracciones de arena, arcilla y limo (Gee y Bouder, 1986), materia orgánica (Nelson y Sommers, 1996), pH (Thomas, 1996), capacidad de intercambio catiónico (CIC) (Sumner y Miller, 1996), y porcentaje de sodio intercambiable (PSI) que se midieron en el extracto de $\mathrm{NH}_{4} \mathrm{OAc}$ $1 \mathrm{M}, \mathrm{pH} 7$, obtenido del desplazamiento de cationes producido en el análisis de CIC. La cuantificación de $\mathrm{Ca}^{2+}, \mathrm{Mg}^{2+}, \mathrm{Na}^{+}$y K+ intercambiables se realizó por espectrofotometría de absorción atómica con un equipo Metrolab 4200. 


\section{Bioensayo de infectividad de los suelos}

Para estimar la infectividad de los suelos de cada sitio, se realizaron tres diluciones de cada suelo proveniente de los cinco árboles (1:0, 1:4 y $1: 40 \mathrm{v} / \mathrm{v})$ en mezcla estéril de perlita: vermiculita (1:1 v/v) (Díaz y Honrubia, 1993). Se utilizó alfalfa (Medicago sativa L.) como planta hospedadora, por su alta capacidad de establecer simbiosis con HMA y producir una respuesta positiva a la inoculación micorrícica. Se sembraron, dos semillas por maceta para cada sitio, teniendo en cuenta los cinco árboles muestreados, las tres diluciones, los tres muestreos y las seis plantas por muestreo: 270 plantas (135 macetas) por cada sitio, 540 plantas en total las cuales fueron alojadas en una cámara de crecimiento en condiciones controladas (16:8 h luz: oscuridad a $25 \pm 2{ }^{\circ} \mathrm{C}$ ) y regadas con agua filtrada. Para observar el estado de micorrización de las raíces se realizaron tres muestreos a los 15, 30 y 60 días de iniciado el ensayo en los que se tomaron para cada tiempo de muestreo: seis plántulas de cada árbol, en las tres diluciones y de los dos sitios (180 plantas totales).

\section{Aislamiento y propagación de los hongos micorrícicos arbusculares en plantas trampa e identificación de esporas en los inóculos}

Para el aislamiento de los HMA se emplearon macetas de $500 \mathrm{~g}$ con una mezcla de $250 \mathrm{~g}$ del suelo de campo de los sitios muestreados (que contienen los tres tipos de propágulos de los HMA: esporas, micelios y raíces colonizadas), y $250 \mathrm{~g}$ de sustrato conformado por una mezcla de tierra: arena: vermiculita: perlita $(1: 1: 1: 1 \mathrm{v} / \mathrm{v})$. El sustrato se tindalizó con vapor de agua durante $1 \mathrm{~h}$, se dejó reposar 24 h, y luego se repitió una vez más toda la operación (Sieverding, 1991). Como planta trampa se empleó alfalfa (Medicago sativa L.), sorgo (Sorghum bicolor (L.) Moench.) y algarrobo blanco ( $P$. alba Griseb.). Las semillas fueron desinfectadas en hipoclorito de sodio $1 \%$ ( $/ / v)$ por 5 min, se enjuagaron con cinco volúmenes de agua, se dejaron sumergidas en agua destilada por $24 \mathrm{~h}$ a temperatura ambiente para su imbibición y luego de germinadas, se colocaron en las macetas. Por cada suelo rizosférico de cada árbol de los dos sitios muestreados se utilizaron 15 macetas, colocando seis plantas por maceta de cada una de las especies utilizadas como planta trampa (150 macetas en total), las cuales fueron cultivadas en invernáculo a $25 \pm 2{ }^{\circ} \mathrm{C}$ y $16: 8 \mathrm{~h}$ luz: oscuridad (intensidad de luz $400 \mu \mathrm{mol} \mathrm{m} \mathrm{m}^{-2} \mathrm{~s}^{-1}$ ) y regadas con agua filtrada para reducir el contenido de cloro. Luego de un año de crecimiento en presencia de los inóculos nativos, las raíces de las plantas trampa fueron colectadas, cortadas y mezcladas con parte del sustrato conteniendo hifas y esporas. A esta mezcla se le adicionó tierra y arena estériles en una relación 3:1:1 (mezcla de inóculo: tierra: arena $v / v$ ) y se mantuvo en las mismas condiciones por un año más. Luego de dos años de mantener las plantas trampa con los inóculos, se tomaron tres submuestras de $50 \mathrm{~g}$ de suelo por sitio y por maceta, para la identificación de las especies de HMA en los inóculos.

\section{Potencial micorrícico de los inóculos de HMA nativos obtenidos de plantas trampa de Colonia Benítez y Padre Lozano}

El potencial micorrícico de los suelos (inóculo, en este trabajo) se estimó según Plenchette, Perrin y Duvert (1989). El potencial micorrícico es la capacidad de iniciar la formación de asociaciones micorrícicas a partir de una cantidad de inóculo, que está formado por esporas, micelio y raíces micorrizadas (Duponnois, Founoune, Masse y Pontanier, 2005)

Esta metodología se basa en una relación dosisrespuesta empleando plántulas de puerro (Allium porrum L.) por su alta capacidad de establecer simbiosis con HMA crecidas durante 28 días en diferentes concentraciones del inóculo y sustrato estéril bajo condiciones controladas.

Las semillas de puerro se desinfectaron con una solución de $\mathrm{H}_{2} \mathrm{O}_{2}$ al $10 \%$ (v/v) por 5 min. Luego se enjuagaron con agua destilada y se sembraron en cámara húmeda en estufa a $25^{\circ} \mathrm{C}$ con luz. A los ocho días se trasplantaron 10 plántulas de puerro por maceta empleándose macetas de $250 \mathrm{~g}$ de capacidad, con los inóculos de ambos sitios en porcentajes 100, 30, 10 y $3 \%$ p/p, completados hasta el $100 \%$ del peso con tierra estéril. Se utilizaron cuatro macetas para cada dilución del suelo (32 macetas totales). Las plántulas fueron mantenidas en una cámara de condiciones controladas (16:8 h luz: oscuridad, a $25 \pm 2{ }^{\circ} \mathrm{C}$ ), regadas con agua filtrada. A los 28 días, se muestrearon 40 plántulas de cada tratamiento y porcentaje de inóculo. Se tiñeron las raíces y la presencia de al menos un punto de entrada o inicio de colonización micorrícica en la raíz se consideró como un registro positivo.

Para el cálculo del potencial micorrícico, se generaron curvas de regresión entre el porcentaje de plantas con presencia de micorrizas en función de la concentración de inóculo, expresado como peso seco de suelo. Mediante la función de regresiones lineales $(Y=a X+b)$ se obtuvo el índice de Infectividad micorrícica del suelo $\left(\mathrm{IMS}_{50}\right.$; inóculo, en este 
trabajo), que es la cantidad de inóculo necesario para infectar el $50 \%$ de las plantas.

\section{Evaluación de la micorrización}

Tanto para el bioensayo de infectividad como para la determinación del potencial de inóculo se tomaron muestras del sistema radical de las plantas, y se tiñeron según una modificación de la metodología propuesta por Phillips y Hayman (1970), en la que se omitió el uso de fenol en los reactivos. Las raíces fueron clarificadas con $\mathrm{KOH} 10 \%(\mathrm{p} / \mathrm{v})$ durante $30 \mathrm{~min}$ a $85{ }^{\circ} \mathrm{C}$; luego se las lavó con agua corriente y para avanzar con el aclaramiento y acidificación las muestras se cubrieron con $\mathrm{HCl} 1 \%(\mathrm{p} / \mathrm{v})$ durante $30 \mathrm{~min}$ temperatura ambiente, se retiró la solución y se les agregó azul de anilina (0,05 \% p/v) en lactoglicerol (ácido láctico: glicerol: agua en proporción 1:1:1 v/v) por 15 min a $90^{\circ} \mathrm{C}$, para su tinción, luego se las enjuagó con abundante agua. Los porcentajes de micorrización fueron determinados según el método de McGonigle, Miller, Evans, Fairchild y Swam (1990) y se observó la posible presencia de otros hongos radicales mediante observación al microscopio óptico Olympus BX40.

\section{Identificación de las especies de HMA de suelos de algarrobales y de los inóculos}

Tanto para la identificación de las especies de HMA de los suelos como para las de los inóculos, se aislaron esporas utilizando el método del tamizado húmedo y decantado (Gerdemann y Nicolson, 1963), seguido de una centrifugación en gradiente de sacarosa al 80 \% (p/v). Las esporas de HMA se montaron en portaobjeto con alcohol polivinílico con y sin agregado de reactivo de Melzer. Posteriormente se examinaron por microscopia óptica y se identificaron morfológicamente por el sistema taxonómico propuesto por Schüler y Walker (2010), por comparaciones con las descripciones de especies publicadas en el sitio web de International Culture Collection of Vesicular Arbuscular Mycorrhizal Fungi (INVAM) y material del cepario del Instituto Spegazzini.

\section{Análisis estadístico}

Los datos obtenidos del análisis de suelo se trataron estadísticamente mediante un análisis de varianza (ANOVA). Los medios ajustados se compararon a posteriori mediante la prueba DGC utilizando Infostat (Di Rienzo et al., 2017). Los datos se presentan como el promedio \pm desvío estándar. Los valores de $\mathrm{p}<0,05$ se consideraron estadísticamente significativos.

\section{RESULTADOS Y DISCUSIÓN}

\section{Análisis físico-químico de los suelos provenientes de sitios de algarrobales bajo condiciones edafoclimáticas contrastantes: húmedo y semiárido del Parque Chaqueño}

Los suelos de CB y PL presentaron una textura franco-arenosa, mostrando similar contenido de magnesio y capacidad de intercambio catiónico del suelo $(\mathrm{ClC})$. Sin embargo, el análisis físicoquímico mostró diferencias significativas entre ambos suelos, en el resto de los parámetros medidos (Tabla 1). El porcentaje de materia orgánica y los contenidos de carbono orgánico y nitrógeno total fueron mayores en $\mathrm{PL}$, con una mayor relación $\mathrm{C} / \mathrm{N}$. Además, el suelo de PL presentó un alto contenido en fósforo e incrementos significativos de calcio y potasio, con un pH ligeramente ácido (Soil Quality Test Kit Guide USDA, 1999). Por su parte, CB mostró mayor contenido en sodio, presentando también mayor porcentaje de sodio intercambiable (PSI) y pH del suelo ligeramente básico.

Factores como la acidez, las concentraciones de materia orgánica, fósforo, nitrógeno, aluminio, cobre y zinc en el suelo, influyen sobre el buen establecimiento y desempeño de la simbiosis con HMA, lo cual se refleja en la capacidad de colonización de hospederos y la producción de esporas de los hongos (Bhatia, Sundari y Adholeya, 1996). En este trabajo, ambos sitios presentaron diferencias edafoclimáticas significativas. Esto sugiere una posible variabilidad en la capacidad de micorrización del suelo entre ambos sitios.

\section{Bioensayo de infectividad con HMA de los suelos provenientes de algarrobales bajo condiciones edafoclimáticas contrastantes}

El bioensayo de infectividad del suelo de CB mostró que a 15 y 30 días de iniciado el tratamiento, el suelo no promovió diferencias de micorrización en las tres diluciones realizadas con un porcentaje de infección cercano al $10 \%$ de las plantas (Figura 2A). A los 60 días se produjo un incremento importante: el suelo sin diluir alcanzó un $35 \%$ de colonización micorrícica y las diluciones 1:4 y 1:40 estimularon la capacidad de micorrización alcanzando alrededor del $70 \%$ de plantas micorrizadas. 
Tabla 1. Características físico-químicas de los sitios de colecta de las muestras de suelos de Colonia Benítez (húmedo) y Padre Lozano (semiárido). PSI, porcentaje de sodio intercambiable; CIC, capacidad de intercambio catiónico del suelo. Letras diferentes indican diferencias significativas (ANOVA, $p<0,05$ ).

\begin{tabular}{lcc}
\hline & Colonia Benítez & Padre Lozano \\
\hline Precipitaciones promedio [mm anuales] & 1300 & 650 \\
Textura & franco arenosa & franco arenosa \\
Materia orgánica [\%] & $2,2 \pm 0,4 \mathrm{~b}$ & $5 \pm 1 \mathrm{a}$ \\
Carbono orgánico [\%] & $1,3 \pm 0,2 \mathrm{~b}$ & $2,9 \pm 0,7 \mathrm{a}$ \\
Nitrógeno total [\%] & $0,12 \pm 0,02 \mathrm{~b}$ & $0,22 \pm 0,05 \mathrm{a}$ \\
Relación C/N & $10,2 \pm 0,4 \mathrm{~b}$ & $11,3 \pm 0,9 \mathrm{a}$ \\
Fósforo [ppm] & $32 \pm 8 \mathrm{~b}$ & $122 \pm 19 \mathrm{a}$ \\
pH & $7,5 \pm 0,4 \mathrm{a}$ & $6,0 \pm 0,3 \mathrm{~b}$ \\
$\mathrm{PSI}$ & $19 \pm 4 \mathrm{a}$ & $2,0 \pm 0,6 \mathrm{~b}$ \\
$\mathrm{CIC}[$ meq / 100 g] & $16 \pm 1 \mathrm{a}$ & $17 \pm 1 \mathrm{a}$ \\
\hline Cationes intercambiables [meq / 100 g] & $15 \pm 2 \mathrm{~b}$ & $21 \pm 2 \mathrm{a}$ \\
$\mathrm{Ca}{ }^{2+}$ & $3 \pm 1 \mathrm{a}$ & $2 \pm 1 \mathrm{a}$ \\
$\mathrm{Mg}^{2+}$ & $5 \pm 2 \mathrm{a}$ & $0,46 \pm 0,06 \mathrm{~b}$ \\
$\mathrm{Na}^{+}$ & $1,3 \pm 0,2 \mathrm{~b}$ & $2,9 \pm 0,6 \mathrm{a}$ \\
$\mathrm{K}^{+}$ & & \\
\hline
\end{tabular}

Por su parte, el bioensayo de infectividad con HMA del suelo puro de PL (Figura 2B) aumentó progresivamente el porcentaje de micorrización alcanzando cerca de 10, 20 y $30 \%$ de plantas micorrizadas a los 15, 30 y 60 días luego de la inoculación, respectivamente. No se observaron diferencias significativas entre las diluciones.

Si se compara la capacidad de micorrización entre CB y PL a los 60 días, se observa que el suelo puro de PL presentó un porcentaje de micorrización similar al suelo puro de CB. Sin embargo, el suelo de CB logró generar el doble de porcentaje de micorrización al ser diluido con sustrato estéril, resultando en una mayor infectividad. Esto sugiere la posible presencia de compuestos tóxicos o inhibidores, que, al ser diluidos, reducen su efecto perjudicial. En estudios realizados con suelos del sur de Canadá, se observaron incrementos en el porcentaje de micorrización en algunas diluciones del inóculo, disminuyendo tanto para diluciones mayores como menores. Este comportamiento se observó en una de las especies vegetales anali-
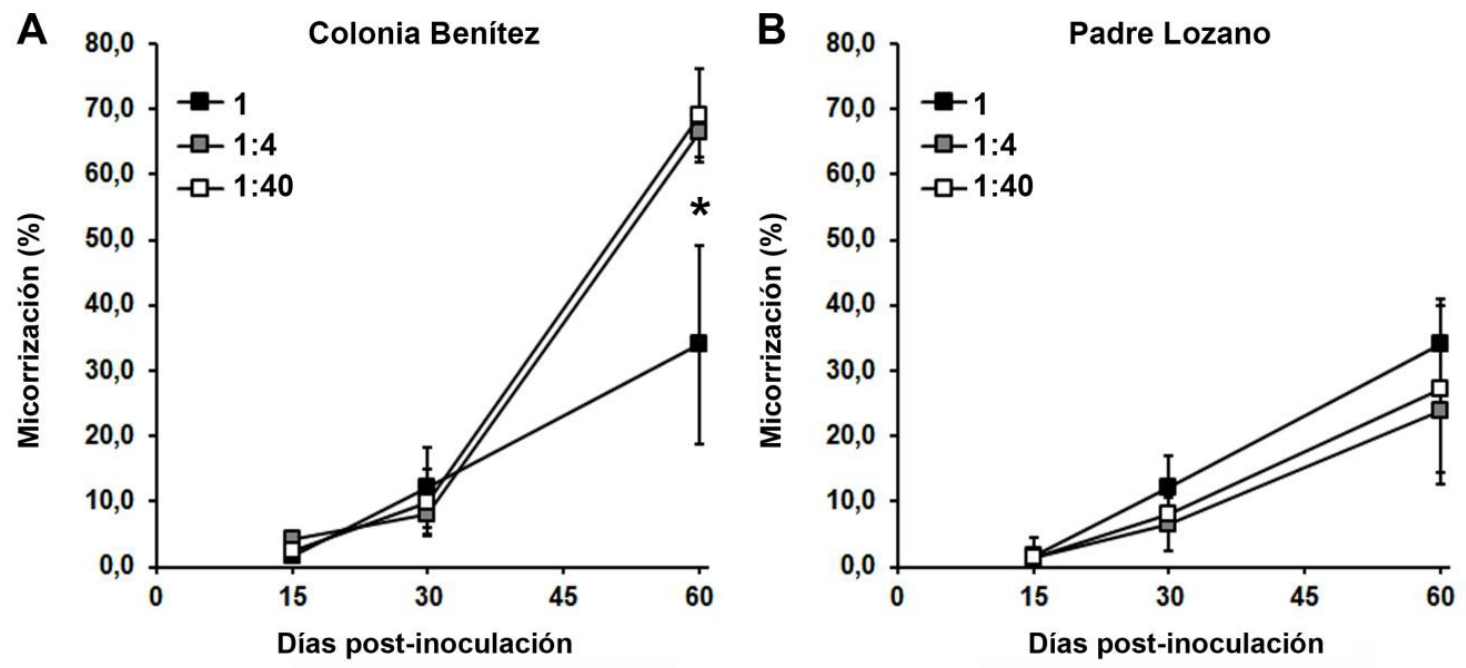

Figura 2. Infectividad de los suelos de Colonia Benítez (A) y Padre Lozano (B). Se muestran los porcentajes de micorrización de las plantas de alfalfa a los 15, 30 y 60 días, tratadas con el suelo puro (1:0), y con diluciones suelo: sustrato estéril (perlita: vermiculita 1:1 $\mathrm{v} / \mathrm{v}$ ) en proporción 1:4 o 1:40 v/v. Los resultados están expresados como promedio \pm error estándar $(\mathrm{n}=5)$. El asterisco indica diferencias significativas (ANOVA, $\mathrm{p}<0.05$ ). 
zadas, y particularmente para la presencia de hifas, pero no en los arbúsculos (Clapperton y Reid, 1992). Esto indica que este tipo de comportamiento en el cual se observa una mayor micorrización con el inóculo diluido, podría depender de la especie vegetal hospedante y su interacción con los HMA. Asimismo, un factor que podría estar inhibiendo la micorrización es el sodio, el cual ha sido reportado ampliamente como un factor que afecta el crecimiento y viabilidad de las hifas (García y Mendoza, 2008). El análisis físico-químico del suelo de algarrobares proveniente de CB mostró pH moderadamente alcalino, asociado con mayores valores de $\mathrm{Na}^{+}$(5,01 meq/ $\left.100 \mathrm{~g}\right)$ y PSI (19,68 \%). Tales características edafológicas podrían explicar el incremento en la colonización micorrícica a medida que el suelo de CB fue diluido. Es conocido que el $\mathrm{pH}$ del suelo tiene un marcado efecto en las comunidades de HMA en agroecosistemas y cultivos (Yang et al., 2013). Las respuestas de los HMA al pH del suelo son variables (Clark, Zeto y Zobel, 1999) encontrándose respuestas positivas de algunos HMA en $\mathrm{pH}$ ácidos y de otros en $\mathrm{pH}$ alcalino, negativas o neutras (Clark et al., 1999).

Por otra parte, un alto contenido de fósforo del suelo también ha sido asociado a las tasas de colonización de raíces y menor diversidad de HMA (Cheng, Ishimoto, Kuriyama, Osaki y Ezawa, 2013). En nuestro estudio encontramos que las menores tasas de colonización micorrícica correspondientes al suelo de PL, estuvieron acompañadas por niveles de fosforo casi cuatro veces mayores a los encontrados en CB. El suelo de PL también presentó mayores niveles de carbono orgánico y nitrógeno total. Altos niveles de N y P en el suelo han sido asociados a bajos niveles de colonización, relacionados con efectos negativos sobre el desarrollo de las micorrizas arbusculares y la estimulación del crecimiento en las plantas (Treseder y Allen, 2002; Alvarado, Chavarría, Guerrero, Boniche y Navarro, 2004). Los niveles de fósforo, tanto los altos como los bajos, y la fertilización nitrogenada, disminuyen el porcentaje de infección de los hongos micorrícicos, mientras que niveles moderados de fósforo incrementan los niveles de nitrógeno y la infección por estos hongos. La forma del nitrógeno inorgánico en el suelo influye en el porcentaje de colonización, la longitud de las raíces y la presencia de estructuras colonizantes, así como los arbúsculos y vesículas (Xin, Jianjun, Guiye y Shuijin, 2005). Asimismo, el suelo de PL presentó mayores valores de calcio y potasio (Tabla 1 y Figura 2). Resultados similares fueron reportados por Pérez, Rojas y Montes (2011) quienes observaron los menores porcentajes de colonización micorrícica en raíces del pasto colosoana, pertenecientes a suelos con valores altos de calcio $(32,2 \mathrm{meq} / 100 \mathrm{~g})$ y potasio (1,3 meq/100 g). En síntesis, la menor infectividad observada en los suelos de PL podría deberse a los mayores niveles de fósforo, nitrógeno total, calcio y potasio, y menor contenido de $\mathrm{Na}^{+}$y PSI.

\section{Potencial micorrícico de los inóculos}

En la Figura 3 se muestra el potencial micorrícico de los inóculos. Sólo en la proporción al 3 \% del inóculo se observaron diferencias significativas (Figura 3A). En esta proporción, el inóculo aislado de CB obtuvo un porcentaje de plántulas infectadas de más del doble con respecto al inóculo de PL. En proporciones mayores de inóculo (30 \% o inóculo puro) ambos produjeron $100 \%$ de plántulas colonizadas.

El desarrollo de una infección micorrícica es afectado por varios factores, el más importante de ellos es el número y tamaño de las estructuras infectivas: hifas, fragmentos de raíces micorrizadas y esporas. Sin embargo, ante una determinada cantidad de inóculo, la estrategia de colonización de las especies es la que define el inicio de la infección radical. Para determinar la infectividad de los inóculos obtenidos de los dos sitios en estudio, y a partir de los porcentajes de plántulas infectadas (Figura 3A), se calculó el coeficiente de regresión entre el porcentaje de plantas con presencia de micorrizas en función de la concentración de inóculo, por lo que se obtuvo el índice de infectividad micorrícica del suelo $\left(\mathrm{IMS}_{50}\right)$ que indica la mínima cantidad de inóculo necesaria para infectar el $50 \%$ de las plántulas (Plenchette, Perrin y Duvert, 1989). Los resultados de la cuantificación de puntos de entrada e hifas intrarradicales, y algunas estructuras de formación temprana como arbúsculos y/o vesículas en las raíces infectadas con los dos inóculos de HMA, muestran una infectividad 3,4 veces mayor en CB que en PL (Figura 3B). Estos resultados indican una diferencia importante en la infectividad de los inóculos provenientes de ambos sitios.

\section{Identificación de las especies de HMA presentes en los suelos y en los inóculos provenientes de CB y PL}

Determinar la presencia de los HMA en función de las esporas resulta ser un método práctico que se ha empleado con éxito en distintos ecosistemas (Kapoor, Sharma y Bhatnagar, 2008) como una aproximación al conocimiento de la integración y funcionalidad de las comunidades de HMA. En la Tabla 2 se registran las especies de HMA identifi- 


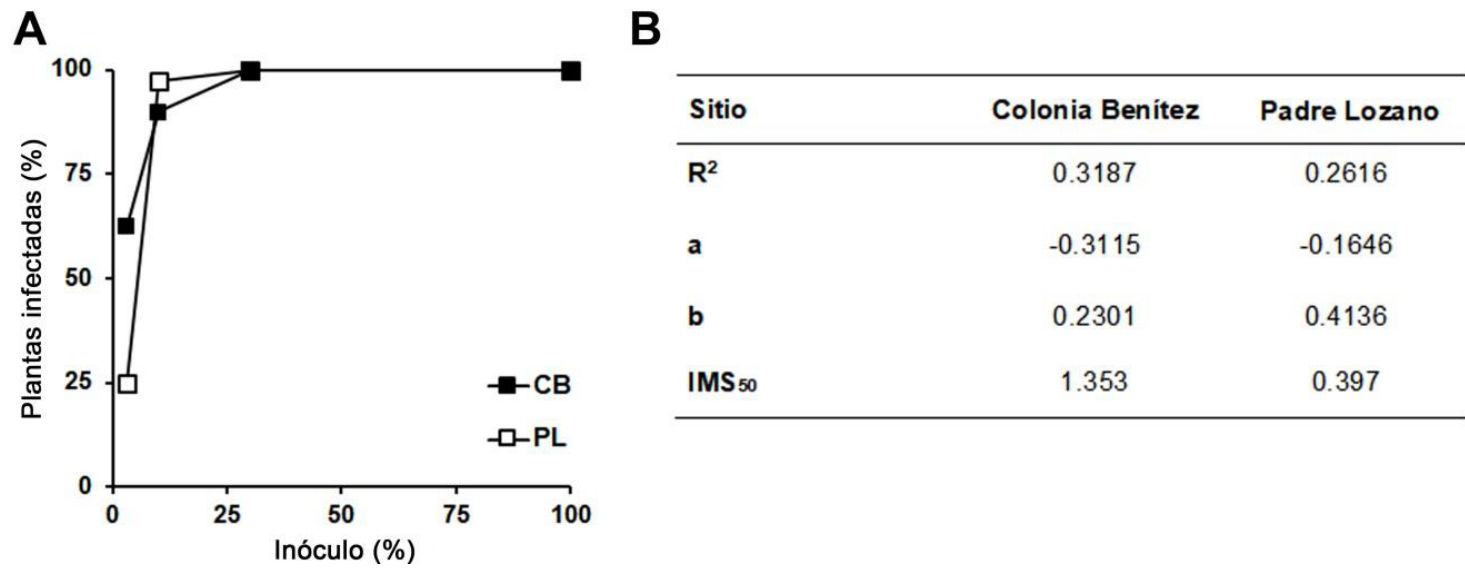

Figura 3. Potencial micorrícico de los inóculos obtenidos de plantas trampa de Colonia Benítez (CB, húmedo) y Padre Lozano (PL, semiárido). A) Porcentaje de micorrización en raíces de puerro. Los cuadrados negros muestran los resultados obtenidos para el sitio CB y los blancos para el sitio PL. Los resultados se expresan como porcentaje de plantas micorrizadas obtenidas en un experimento representativo. B) Coeficientes de regresión y estimación del índice $\mathrm{IMS}_{50}$ a partir de bioensayo mostrado en (a). IMS $\mathrm{IS}_{50}$ indica la mínima cantidad de inóculo en gramos por cada $100 \mathrm{~g}$ de suelo seco, necesaria para infectar el 50 \% de las plantas.

cadas morfo-taxonómicamente a partir del análisis de las esporas presentes en los suelos de ambos sitios. En general, las esporas observadas fueron pequeñas y escasas. En el sitio de CB, se observaron al menos cuatro especies de HMA; en PL sólo se identificó una especie y se encontraron dos especies no identificadas de Glomus; mientras Acaulospora laevis fue identificada en ambos sitios.

Dado que una alta proporción de HMA produce esporas estacionalmente (Brundrett y Ashwath, 2013), y que la especie vegetal puede influir en la diversidad de especies de HMA que proliferan en el suelo, la estimación de la diversidad también se realizó de forma complementaria en los inóculos propagados en plantas trampas de sorgo, alfalfa y algarrobo a partir de las muestras de suelos de los sitios húmedo y semiárido. En la Tabla 3, se muestran las HMA identificadas mediante el análisis de las esporas presentes en el sustrato. En ambos sitios se observaron tres especies compartidas:
F. mosseae, R. intraradices y C. etunicatum; dos especies exclusivas de CB, $S$. constrictum y $R$. clarus; y dos especies exclusivas de PL, C. claroideum y $D$. spurca.

La única especie encontrada en ambos inóculos que coincidió con las especies encontradas en suelo (sólo en el suelo de CB) fue $R$. intraradices. Las demás especies fueron identificadas en uno o en ambos sitios, pero exclusivamente en suelos (Tabla 2) o en inóculos (Tabla 3). Estos resultados reafirman la necesidad de complementar la identificación de HMA en suelo con su propagación mediante plantas trampa, que permitan identificar HMA con bajo número de esporas en los suelos.

La presencia predominante de la familia Glomeraceae hallada en este estudio coincide con lo observado en ecosistemas semiáridos de Brasil (de Souza, Rodríguez-Echeverría, Andrade y Freitas, 2016) y en un estudio de la influencia del pastoreo en plantas del desierto en el cual se

Tabla 2: Identificación morfo-taxonómica de las especies de HMA de los suelos de Colonia Benítez (húmedo) y Padre Lozano (semiárido). (+) Indica presencia y (-) indica ausencia.

\begin{tabular}{|c|c|c|c|}
\hline Familia & Especie & Colonia Benítez & Padre Lozano \\
\hline \multirow[t]{4}{*}{ Glomeraceae } & Glomus sp. 1 & - & + \\
\hline & Glomus sp. 2 & - & + \\
\hline & Funneliformis geosporum & + & - \\
\hline & Rhizophagus intraradices & + & - \\
\hline Acaulosporaceae & Acaulospora laevis & + & + \\
\hline Entrophosporaceae & Entrophospora nevadensis & + & - \\
\hline
\end{tabular}


Tabla 3. Identificación morfo-taxonómica de las especies de HMA de Colonia Benítez (húmedo) y Padre Lozano (semiárido) aislados y propagados dos años en plantas trampas. (+) Indica presencia y (-) indica ausencia.

\begin{tabular}{llcc}
\hline Familia & Especie & Colonia Benítez & Padre Lozano \\
\hline & Funneliformis mosseae & + & + \\
& Septoglomus constrictum & + & - \\
& Rhizophagus intraradices & + & + \\
& Rhizophagus clarus & + & + \\
Claroideoglomeraceae & Claroideoglomus etunicatum & + & + \\
& Claroideoglomus claroideum & - & + \\
Diversisporaceaea & Diversispora spurca & - & + \\
\hline
\end{tabular}

reportó una diversidad de 13 especies, la mayoría de ellas de la familia Glomeraceae ( $\mathrm{Ba}$ et al., 2012). La identificación de familias de HMA como Glomeraceae y Claroideoglomeraceae coincide con estudios realizados en pastizales semiáridos (Xu et al., 2017). Los miembros de la familia Glomeraceae son capaces de producir grandes cantidades de esporas en períodos cortos de tiempo y regular su crecimiento como parte de las funciones como hospedante. Estas características corresponden a especies con estrategias de tipo R, generalistas y con gran producción de propágulos que garantizan la supervivencia de la especie en el sitio. Estas especies suelen ser dominantes en áreas perturbadas de diversos ambientes dominantes desde estepas (Ba et al., 2012), praderas de pastizales (Stover, Thorn, Bowles, Bernards y Jacobs, 2012) y bosques de sabanas, hasta humedales y selvas (Brundrett y Ashwath, 2013). Dentro de la familia Glomeraceae, en este estudio se ha identificado especies de HMA como C. etunicatum, F. mosseae y R. intraradices, frecuentemente reportadas en asociación con la vegetación de ecosistemas áridos y semiáridos de Estados Unidos (Stutz, Copeman, Martin y Morton, 2000), México (Montaño et al., 2012) y África (Uhlmann, Görke, Petersen, Oberwinkler, 2006). El género Rhizophagus presenta gran tolerancia al estrés ambiental y al pastoreo (Yang et al., 2013), mientras Glomus se adapta bien a hospedantes de diversas calidades, demostrando suficiente capacidad de establecer relaciones simbióticas con numerosas especies vegetales (Cai et al., 2014).

Como aspecto de interés, en el inóculo aislado de planta trampa de $\mathrm{CB}$, se identificó $F$. geosporum que es una especie de hongo que produce muchas esporas de manera sostenida, fenómeno que le permite tener una distribución temporal amplia dentro del ecosistema. Adicionalmente, se ha reportado que esta especie de HMA se adapta fácilmente a diferentes condiciones ambientales y a sus cambios bruscos (He et al., 2014). Las especies $A$. laevis y $F$. geosporum coinciden con los reportes realizados por Lara (2004), que encontró estas especies de HMA en suelos de huertas de aguacate. F. mosseae, ha sido reportada en una amplia gama de ecosistemas terrestres y se caracteriza por su ubicuidad (Oehl et al., 2012). Esta especie ha logrado sobreponerse exitosamente a las prácticas agrícolas relacionadas con la producción de soja, tales como el uso de pesticidas y la fertilización, las cuales afectan negativamente a ciertas especies de Glomeromycota (Cabello, 2013) y puede aprovechar las tres fuentes de inóculos: posee elevada producción de esporas, las hifas son altamente infectivas incluso en situaciones de déficit hídrico y forma vesículas intrarradicales que les permite iniciar nuevas infecciones (Biermann y Linderman, 1983). Sin embargo, ha resultado sensible a altas presiones de pastoreo, de forma similar a $D$. spurca, que tolera bien condiciones de sequía (Soteras et al., 2013). Algunas especies del género Diversispora predominan en ambientes ricos en humus, indicando que este es frecuente en suelos de bajo historial de uso antrópico (Liu et al., 2015). También se encontró gran abundancia de Diversisporales en suelos de una reserva natural tropical (Saks et al., 2013; Camenzind et al., 2014).

C. etunicatum ha sido encontrado en áreas degradadas (Cabello, 2013), y resulta muy eficiente cuando las plantas están expuestas a condiciones de altos niveles de estrés ambiental. $R$. intraradices es una especie considerada nitrofílica por lo que se le encuentra con mayor frecuencia asociada a plantas leguminosas con alto contenido de nitrógeno (Barrer, 2009); en los suelos de algarrobales analizados en este trabajo, ha sido identificada en CB cuando se analizaron los suelos (Tabla 2) y en ambos sitios cuando se analizaron los inóculos (Tabla 3). Congruentemente en este trabajo, sólo fue identificado en los inóculos generados con suelo de PL, que posee el doble de materia orgánica y carbono orgánico que $\mathrm{CB}$, 
aunque ambos son de textura franco-arenosa.

Es interesante destacar que un mayor nivel de fósforo en el suelo se asocia con una menor tasa de colonización de micorrizas en las raíces y una menor diversidad y abundancia de los HMA, siendo esta última influida positivamente por el contenido de nitrógeno (Koorem et al., 2014). En estosresultados, los valores de fósforo y nitrógeno total en el suelo fueron considerablemente contrastantes entre CB y PL (Tabla 1), y no se encontraron diferencias en la presencia de especies ni de géneros, aunque existen algunas familias que se encontraron exclusivamente en uno de los sitios y podrían estar influidas por estos componentes de los suelos, como son E. nevadensis (en CB) y D. spurca (en PL) (Tabla 2 y 3 ).

La riqueza de especies de HMA en cien sitios representativos de los matorrales xerófilos del Valle del Mezquital resultó alta (29 morfo-especies) en comparación con lo que se registró en comunidades áridas de Chihuahua (24 especies) (Montaño et al., 2012) en México; y en las selvas tropicales Iluviosas de China (27 especies) (Zhao et al., 2003). En este trabajo se reporta una riqueza de especies aun menor (ocho especies en cada sitio, considerando tanto las especies identificadas del suelo como del inóculo), similar a lo reportado por Lopes Leal, Stürmer y Siqueira (2009) en un análisis comparativo entre diversos ecosistemas, desde pasturas hasta bosques, en el que reportan entre 5 y 14 especies por ecosistema; similar también a la riqueza observada en el Parque Nacional de las Sierras de las Quijadas, San Luis, Argentina (Rivero Mega, Crespo, Molina y Lugo, 2014), coincidiendo en este último caso sólo en la especie $F$. geosporum. Esta diferencia en la riqueza de especies podría estar influenciada no sólo por las características edafoclimáticas, sino también por otros factores que no han sido analizados en estos trabajos, como la profundidad y usos del suelo y la diversidad de especies vegetales. Además, en aquellos trabajos en donde se reporta un alto número de especies, las diferencias en el número de estas también podrían deberse a que se reporta la riqueza de especies total de una gran área de muestreo, lo cual dificulta la comparación entre sitios.

\section{CONCLUSIÓN}

Este es el primer estudio en Argentina en el que se analiza la diversidad de HMA en suelos de algarrobales y se mide la infectividad de los suelos y de los inóculos obtenidos a partir de ellos. Los sitios de Colonia Benítez (húmedo) y Padre Lozano (semiárido) presentaron diferencias edáfi- cas significativas y esto fue acompañado de una variabilidad en la infectividad y diversidad de los HMA aislados. El sitio de PL tuvo las menores precipitaciones y el fósforo fue abundante en el suelo, presentando mayor acidez que el suelo de CB, lo cual se correspondió con una menor capacidad infectiva y menor potencial micorrícico del inóculo de HMA, comparado con CB. Una especie propia de ambientes áridos como Acaulospora laevis se observó en ambos sitios. También, hubo diferentes especies de HMA según el sitio de aislamiento. En el sitio de CB se identificaron HMA que se adaptan a diferentes condiciones ambientales como Funneliformis geosporum, Rhizophagus intraradices, Rhizophagus clarus y Entrophospora nevadensis, mientras que en plantas trampas de PL se identificó Diversispora spurca, propia de suelos con alto contenido orgánico y tolerante a la sequía. Actualmente se realizan comparaciones del efecto de ambos inóculos en simbiosis con $P$. alba bajo condiciones de riego y resultados preliminares señalan al inóculo de CB con un efecto positivo sobre el desarrollo de plantas de $P$. alba micorrizadas, que pudo observarse en un mayor número de hojas.

\section{AGRADECIMIENTOS}

Este trabajo fue financiado por los proyectos PROMEF, Proyecto Nacional Forestales, INTA (PNFOR-1104073) y Agencia Nacional de Promoción Científica y Tecnológica (PICT 2012-0339). La Ing. Agr. Mónica B. Sagadin fue financiada por una beca de formación INTA (Res. 516/07 y 935/10). Los autores agradecen a Aníbal Verga por su colaboración proveyendo infraestructura e insumos para este trabajo; a Carlos Gómez, Sebastián Kees y Diego López Lauenstein por el muestreo de los suelos, y a Agustín Carbajal por la lectura crítica del manuscrito. Los autores declaran no tener ningún conflicto de intereses y no haber realizado experimentos en seres humanos ni animales.

\section{BIBLIOGRAFÍA}

Alvarado, A., Chavarría, M., Guerrero, R., Boniche, J. y Navarro, J. (2004). Características edáficas y presencia de micorrizas en plantaciones de teca (Tectona grandis) en Costa Rica. Agronomía Costarricense, 28 (1), 89-100. ISSN 0377-9424.

Arévalo Hernández, C. O. (2016). Prospección de la densidad de esporas y colonización de micorrizas en cacao silvestre de Ucayali y Madre de Dios. Tesis para optar el título de Ingeniero Agrónomo, Universidad Nacional Agraria La Molina, Facultad de Agronomía, 
Lima, Perú. http://repositorio.lamolina.edu.pe/handle/ UNALM/1968

Ba, L., Ning, J., Wang, D., Facelli, E., Facelli, J. M., Yang, Y. y Zhang, L. (2012). The relationship between the diversity of arbuscular mycorrhizal fungi and grazing in a meadow steppe. Plant and Soil, 352, 143-156. doi: 10.1007/s11104-011-0985-6. Online ISSN 1573-5036

Barrer, S. E. (2009). El uso de hongos micorrízicos arbusculares como una alternativa para la agricultura. Facultad de Ciencias Agropecuarias 124 Vol. 7 No. 1 Enero - Junio, Colombia. 7 (1), 123-133. http://www. scielo.org.co/pdf/bsaa/v7n1/v7n1a14.pdf

Becerra, A. y Cabello, M. (2008). Hongos micorrícico arbusculares presentes en bosques de Alnus acuminata Betulaceae de la Yunga Argentina. Boletín de la Sociedad Argentina de Botánica, 43(3-4), 197-203. Versión On-line ISSN 1851-2372

Bhatia N. P., Sundari K., Adholeya A. (1996). Diversity and selective dominance of vesicular-arbuscular mycorrhizal fungi. En Mukerji, K. G. (Ed.) Concepts in Mycorrhizal Research. Handbook of Vegetation Science, vol. 19/2. Springer, Dordrecht. doi: 10.1007/978-94-0171124-1_6.

Biermann, B., Linderman, R. (1983). Use of Vesicular-Arbuscular Mycorrhizal Roots, Intraradical Vesicles and Extraradical Vesicles as Inoculum. The New Phytologist, 95 (1), 97-105. doi: 10.1111/j.1469-8137.1983. tb03472.x

Bray, R. H. y Kurtz, L. (1945). Determination of total, organic, and available forms of phosphorus in soils. Soil Science, 59 (1), 39-46. doi: 10.1097 / $00010694-$ 194501000-00006

Bremner, J. M. (1965). Nitrogen availability indexes. En Black, C. A., et al. (Eds.). Methods of soil analysis. Part 2. Agron. Monogr. 9. ASA and SSSA, Madison, Wisconsin, EEUU. p. 1324-1345.

Brundrett, M. C. y Ashwath, N. (2013). Glomeromycotan mycorrhizal fungi from tropical Australia III. Measuring diversity in natural and disturbed habitats. Plant and Soil, 370, 419-433. doi: 10.1007/s11104-013-1613-4.

Cabello, M. (2013). Biodiversidad de hongos formadores de micorrizas arbusculares reportadas en Argentina. Microbiología Agrícola: un aporte de la investigación en la Argentina (2 ed.). Tucumán, Argentina: Magna Publicaciones. https://www.researchgate.net/publication/282849782.

Cabrera, A. L. (1994). Regiones fitogeografías argentinas. En: Kugler WF (Ed) Enciclopedia argentina de agricultura y jardinería. Tomo 2. $2^{\circ}$ edición. Cumbre. Buenos Aires, Argentina. Fascículo 1. pp. 1-85.

Cai, X. B., Peng, Y. L., Yang, M. N., Zhang, T. y Zhang, Q. (2014). Grassland Degradation Decrease the Diversity of Arbuscular Mycorrhizal Fungi Species in Tibet Pla- teau. Notulae Botanicae Horti Agrobotanici Cluj-Napoca, [S.I.], v. 42, n. 2, p. 333-339. ISSN 1842-4309. doi: 10.15835/nbha4229458

Camardelli, C., Kirschbaum, P., Kirschbaum, A. y Fabrezi, M. (2015). Monitoreo de la degradación de tierras en un área del Chaco Semiárido de la provincia de Salta. Temas de Biología y Geología del NOA. Revista de Divulgación Científica del Instituto de Bio y Geociencias. 5, 38-41. ISSN 1853-6700.

Camenzind, T., Hempel, S., Homeier, J., Horn, S., Velescu, A., Wilcke, W. y Rillig, M. C. (2014). Nitrogen and phosphorus additions impact arbuscular mycorrhizal abundance and molecular diversity in a tropical montane forest. Global Change Biology, 20, 3546-3659. doi: $10.1111 / \mathrm{gcb} .12618$

Cheng, Y., Ishimoto, K., Kuriyama, Y., Osaki, M. y Ezawa, T. (2013). Ninety-year-, but not single, application of phosphorus fertilizer has a major impact on arbuscular mycorrhizal fungal communities. Plant Soil, 365, 397407. doi: 10.1007/s11104-012-1398-X

Chen, C., Jianjun, T., Guiye, Z. y Shuijin, H. (2005). Arbuscular mycorrhizal colonization and phosphorus acquisition of plants: effects of coexisting plant species. Applied Soil Ecology, 28, 259-269. doi: 10.1016/j. apsoil.2004.07.009

Clapperton, M. J. y Reid, D. M. (1992). A relationship between plant growth and increasing VA mycorrhizal inoculum density. The New Phytologist, 120, 227-234. doi: 10.1111/j.1469-8137.1992.tb05658.x

Clark, C. A., Zeto, S. K. y Zobel, R. W. (1999). Arbuscular mycorrhizal fungal isolate effectiveness on growth and root colonization of Panicum virgatum in acidic soil. Soil Biology and Biochemistry, 31, 1757-1763. doi: 10.1016/S0038-0717(99)00084-X

Covacevich, F., Marino, M. A., Echeverria, H. E. (2006). The phosphorus source determines the arbuscular mycorrhizal potential and the native mycorrhizal colonization of tall fescue and wheatgrass in a moderately acidic Argentinean soil. European Journal of Soil Biology, 42, 127-38. doi: 10.1080 / 17429140802546060

de Souza, T. A. F., Rodríguez-Echeverría, S., Andrade, L. A. y Freitas, H. (2016). Arbuscular mycorrhizal fungi Mimosa tenuiflora (Willd.) Poir from Brasilian semi-arid. Brazilian Journal of Microbiology, 47, 359-366. doi: 10.1016 / j.bjm.2016.01.023

Diaz, G. y Honrubia, M. (1993). Infectivity of mine soils from Southeast Spain. Mycorrhiza, 4, 85-88. doi: 10.1007/BF00204063

Di Rienzo, J. A., Casanoves, F., Balzarini, M. G., González, L., Tablada, M. y Robledo, C. W. (2017). Infostat. Córdoba, Argentina: FCA, Universidad Nacional de Argentina. www.infostat.com.ar

Duponnois, R., Founoune, H., Masse, D. y Pontanier, R. 


\section{(2005). Inoculation of Acacia}

holosericea with ectomycorrhizal fungi in a semiarid site in Senegal: growth response and influences on the mycorrhizal soil infectivity after 2 years' plantation. Forest Ecology and Management, 207, 351-362. doi: 10.1016/j.foreco.2004.10.060

García, I. V. y Mendoza, R. E. (2008). Relationships among soil properties, plant nutrition and arbuscular mycorrhizal fungi-plant symbioses in a temperate grassland along hydrologic, saline and sodic gradients. FEMS Microbiology Ecology, 63, 359-371. doi: 10.1111 / j.1574-6941.2008.00441.x

Gee, G. W. y Bauder, J. W. (1986). Particle Size Analysis. En A. Klute (Ed.), Methods of Soil Analysis, 2 ed., vol. 9 (383-411). Madison, Wisconsin, EUA: Am. Soc. Agron.

Gerdemann, J. W. y Nicolson T. H. (1963) Spores of mycorrhizal Endogone species extracted from soil by wet sieving and decanting. Transactions of the British Mycological Society, 84, 679-684. doi: 10.1016/S00071536(63)80079-0

He, L., Yang, H., Yu, Z., Tang, J., Xu, L. y Chen, X. (2014). Arbuscular mycorrhizal fungal phylogenetic groups differ in affecting host plants. Ecology, 80, 1142-1149. doi: 10.1016 / j.jes.2014.07.013

Herrera-Peraza, R.A., Hamel, C., Fernández, F., Ferrer, R.L., Furrazola, E. (2011). Soil-strain compatibility: the key to effective use of arbuscular mycorrhizal inoculants? Mycorrhiza, 21, 183-193. doi: 10.1007 / s00572010-0322-6

Hijmans, R. J., Cameron, S. E., Parra, J. L., Jones, P. G. y Jarvis, A. (2005). Very high resolution interpolated climate surfaces for global land areas. International Journal of Climatology, 25, 1965-1978. doi: 10.1002/ joc. 1276

INVAM, International Culture Collection of Vesicular Arbuscular Mycorrhizal Fungi. Recuperado de: http:// invam.caf.wvu.edu/

Kapoor, R., Sharma, D. y Bhatnagar, A. K. (2008). Arbuscular mycorrhizae in micropropagation systems and their potential applications. Scientia Horticulturae, 116, 227-239. doi: 10.1016/j.scienta.2008.02.002

Koorem, K., Gazol, A., Öpik, M., Moora, M., Saks, Ü., Uibopuu, A. y Sõber, Z. (2014). Soil Nutrient Content Influences the Abundance of Soil Microbes but Not Plant Biomass at the Small-Scale. PLoS ONE, 9 (3), e91998. doi: 10.1371/journal.pone.0091998

Lara, B. N. (2004). Exploración de hongos vesículo-arbusculares en el cultivo de aguacate de Estado de Michoacán. En M. C., González-Chávez, J., Pérez-Moreno, R., Ferrera-Cerrato, M. P., Ortega-Larrocea, Y., Carreón-Abud, y E., Valencia-Cantero (compiladores). Resúmenes del Simposium Nacional y II Simposium Iberoamericano de la Simbiosis Micorrízica. Morelia,
Michoacán, México. https://www.biologicas.umich. $\mathrm{mx} /$ index.php/view/BiologicasVol18No2_2106_1-9

Liu, Y., Johnson, N. C., Mao, L., Shi, G., Jiang, S., Ma, X., Du, G., An, L. y Feng, H. (2015). Phylogenetic structure of arbuscular mycorrhizal community shifts in response to increasing soil fertility. Soil Biology and Biochemistry, 89, 196-205. doi: 10.1016/j.soilbio.2015.07.007

Lopes Leal, P., Stürmer, S. L. y Siqueira, J. O. (2009). Occurrence and diversity of arbuscular mycorrhizal fungi in trap cultures from soils under different land use systems in the Amazon, Brazil. Brazilian Journal of Microbiology, 40, 111-121. doi: 10.1590 / S1517838220090001000019

López Lauenstein, D., Fernández, M. E. y Verga, A. (2012). Respuesta diferenciada a la sequía de plantas jóvenes de Prosopis chilensis, P. flexuosa y sus híbridos interespecíficos: implicancias para la reforestación en zonas áridas. Ecología Austral, 22, 43-52. Versión On-line ISSN 1667-782X

McGonigle, T. P., Miller, M. H., Evans, D. G., Fairchild, G. L. y Swam, J. A. (1990). A new method which gives an objective measure of colonization of roots by vesicular-arbuscular mycorrhizal fungi. The New Phytologist, 115, 495-501. doi: 10.1111/j.1469-8137.1990. tb00476.x

Maherali, H. y Klironomos, J. N. (2007). Influence of phylogeny on fungal community assembly and ecosystem functioning. Science, 316, 1746-1748.

doi:10.1126 / science.1143082

Montaño, A. N. M., Alarcón, A., Camargo-Ricalde, S. L., Hernández-Cuevas, L. V., Álvarez-Sánchez, J., González-Chávez, M. C, Gavito, M., Sánchez-Gallen, I., Ramos-Zapata, J., Guadarrama, P., Maldonado-Mendoza, I. E., Castillo, S., García-Sánchez, R., Trejo, D. y Ferrera-Cerrato, R. (2012). Research on arbuscular mycorrhizae in Mexico: an historical synthesis and future prospects. Symbiosis, 57, 111-126. doi: 10.1007/ s13199-012-0184-0

Nelson, D. y Sommers, J. (1996). Total carbon, organic carbon, and organic matter. En D. L. Sparks, A. L. Page, P. A. Helmke, R. H. Loeppert, P.N. Soluanpour, M. A. Tabatabai, C. T. Johnston y M. E. Sumner (Ed.). Methods of soil analysis part 3: Chemical methods (961-1010). Madison, Wisconsin, EUA: Soil Science Society of America, Inc. and American Society of Agronomy, Inc.

Oehl, F., Laczko, E., Bogenrieder, A., Stahr, K., Bösch, R., van der Heijde, M. y Sieverding, E. (2010). Soil type and land use intensity determines the composition of arbuscular mycorrhizal fungal communities. Soil Biology and Biochemistry, 42, 724-738. doi: 10.1016/j. soilbio.2010.01.006

Pérez, C. A., Rojas, J. S. y Montes, D. V. (2011). Hongos formadores de micorrizas arbusculares: Una alternati- 
va biológica para la sostenibilidad de los agroecosistemas de praderas en el Caribe colombiano. Revista Colombiana de Ciencias Animales, 3 (2), 366-385. ISSN-e 2027-4297.

Phillips, J. M. y Hayman, D. S. (1970). Improved procedures for clearing roots and staining parasitic and vesicular-arbuscular mycorrhizal fungi for rapid assessment to infection. Transactions of the British Mycological Society, 55, 158-161. doi: 10.1016/S00071536(70)80110-3

Piatti, C. L., Iglesias, M. C. (2004). Registro y cuantificación de la presencia de micorrizas vesículo-arbusculares en suelos de algarrobales. Comunicaciones Científicas y Tecnológicas. Resumen: A-058. www.unne. edu.ar/unnevieja/Web/cyt/com2004/5-Agrarias/A-058. pdf

Plenchette, C., Perrin, R. y Duvert, P. (1989). The concept of soil infectivity and a method for its determination as applied to endomycorrhizas. Canadian Journal of Botany, 67, 112-115. doi: 10.1139/b89-016

Rivero Mega, M. S., Crespo, E. M., Molina, M. G. y Lugo, M. A. (2014). Diversidad diferencial de esporas de Glomeromycota en la rizosfera de bromeliáceas nativas del parque nacional sierra de las Quijadas (San Luis, Argentina). Boletín de la Sociedad Argentina de Botánica., 49, 317-325. doi: 10.31055/1851.2372.v49. n3.9462

Rodríguez, G. O. (2018). Colonia Benítez, Reserva Natural Estricta, Chaco. Recuperado de: http://www.patrimonionatural.com/HTML/provincias/chaco/coloniabenitez/coloniabenitez.asp (última visita el 13/03/2018).

Saks, Ü., Davidson, J., Öpik, M., Vasar, M., Moora, M. y Zobel, M. (2013). Root-colonizing and soil-borne communities of arbuscular mycorrhizal fungi in a temperate forest and understory. Botany, 92 (4), 277-285. doi: 10.1139/cjb-2013-0058

Salifu, K. F. y Jacobs, D. F. (2006). Characterizing fertility targets and multi-element interactions in nursery culture of Quercus rubra seedlings. Annals of Forest Science, 63, 231-237. doi: 10.1051/forest: 2006001

Schüßler, A. y Walker, C. (2010). Glomeromycota: a species list with new families and new genera. Recuperado de: http://schuessler.userweb.mwn.de/amphylo/ (última visita el 13/3/2018).

Sieverding, E. (1991). Vesicular arbuscular mycorrhiza in tropical agrosystem. Deutsche Gesellsschaft fur technische Zusammenarbeit GTZ. Eschborn, Germany: $\mathrm{GMBH}$.

Soteras, F., Cofré, N., Bartoloni, J., Cabello, M. y Becerra, A. (2013). Hongos arbusculares (Glomeromycota) en la rizosfera de Atriplex lampa en dos ambientes salinos de Córdoba: influencia de la profundidad en la colonización radical y presencia de morfoespecies. Boletín de la Sociedad Argentina Botánica, 48, 211-

\section{9. doi: 10.31055/1851.2372.v48.n2.6206}

Soil Quality Test Kit Guide USDA, 1999. Recuperado de: https://www.nrcs.usda.gov /Internet/ FSE_ DOCUMENTS/ stelprdb1044790.pdf.

Stover, H. J., Thorn, R. G., Bowles, J. M., Bernards, M. A. y Jacobs, C. R. (2012). Arbuscular mycorrhizal fungi and vascular plant species abundance and community structure in tallgrass prairies with varying agricultural disturbance histories. Applied Soil Ecology, 60, 61-70. doi: 10.1016/j.apsoil.2012.02.016

Stutz, J. C., Copeman, R., Martin, C. A. y Morton, J. B. (2000). Patterns of species composition and distribution of arbuscular mycorrhizal fungi in arid regions of southwestern North America and Namibia, Africa. Canadian Journal of Botany, 78, 237-245. doi: 10.1139/ b99-183

Sumner, M. E y W. P. Miller (1996). Ammonium Acetate $(\mathrm{pH} 7)$ Method. Cation Exchange. Capacity and Exchange Coefficients. Chapter 40. SSSA BOOK SERIES: 5. Methods of soil Analysis. Part 3- Chemical Methods., p 1220.

Tapia-Goné, J., Ferrera-Cerrato, R., Varela-Fregoso, L., Rodriguez-Ortiz, J., Soria- Colunga, J., Tiscareño-Iracheta, M., Loredo-Osti, C., Alcalá-Jáuregui, J. y VillarMorales, C. (2010). Infectividad y efectividad de hongos micorrízicos arbusculares nativos de suelos salinos en el cultivo de lechuga (Lactuca sativa). Revista Mexicana de Micología, 31, 69-74. ISSN 0187-3180

Thomas, G. W. (1996). Soil pH and Soil Acidity. En D. L. Sparks, (Ed.). Methods of Soil Analysis Part 3: Chemical Methods (475-490). Madison, Wisconsin, USA: SSSA Book Series 5, Soil Science Society of America.

Trejo, D., Ferrera, R., García, R., Varela, L., Lara, L. y Alarcón, A. (2011). Efectividad de siete consorcios nativos de hongos micorrízicos arbusculares en plantas de café en condiciones de invernadero y campo. Revista Chilena de Historia Natural, 84, 23-31. ISSN 0716078X

Treseder, K. K. y Allen, M. F. (2002). Direct nitrogen and phosphorus limitation of arbuscular mycorrhizal fungi: a model and field test. The New Phytologist, 155, 507515. doi: 10.1046/j.1469-8137.2002.00470.x

Uhlmann, E., Görke, C., Petersen, A. y Oberwinkler, F. (2006). Arbuscular mycorrhizae from arid parts of Namibia. Journal of Arid Environments, 64, 221-237. doi: 10.1016 / j.jaridenv.2005.05.002

Walkley, A. y Black, I. A. (1934). An examination of the Degtjareff method for determining soil organic matter, and a proposed modification of the chromic acid titration method. Soil Science, 37, 29-38. doi: 10.1097/00010694-193401000-00003

Walker, C., Mize, W. y McNabb, H. S. (1982). Populations of endogenous fungi at two populations in central 
lowa. Canad. J. Bot., 60, 2518-2529. doi: 10.1139/ b82-305

Xu, X., Chen, C., Zhang, Z., Sun, Z., Chen, Y., Jiang, J. y Shen, Z. (2017). The influence of environmental factors on communities of arbuscular mycorrhizal fungi associated with Chenopodium ambrosioides revealed by MiSeq sequencing investigation. Science Reports, 7. 45134. doi: 10.1038/srep45134

Yang, W., Zheng, Y., Gao, C., He, X., Ding, Q., Kim, Y., Rui, Y., Wang, S. y Guo, L. (2013). The Arbuscular
Mycorrhizal Fungal Community Response to Warming and Grazing Differs between Soil and Roots on the Qinghai-Tibetan Plateau. PLoS ONE, 8, e76447. doi: 10.1371/journal.pone.0076447

Zhao, L., Versaw, W. K., Liu, J. y Harrison, M. J. (2003). A phosphate transporter from Medicago truncatula is expressed in the photosynthetic tissues of the plant and located in the chloroplast envelope. The New Phytologist, 157, 291-302. doi: 10.1046/j.14698137.2003.00677.x 